\title{
The Analgesic Effect of Abobotulinum and Incobotulinum Toxins Type A in Central Poststroke Pain: Two Case Reports
}

\author{
Alexandre Camoes-Barbosa, MD, Ana-Filipa Neves, MD
}

\section{Introduction}

The International Association for the Study of Pain defines central pain as "pain initiated or caused by primary lesion or dysfunction of the central nervous system" [1]. If it results from a stroke, it is referred to as central poststroke pain (CPSP) [1]. CPSP prevalence reports vary from $8 \%$ to $46 \%$ [2-4]. This wide variation is possibly related to the heterogeneity of stroke lesions between the populations surveyed, differences in study design, and different timing of evaluations after stroke [5]. CPSP continues to be an under-recognized complication despite its potential to impair activities of daily living ( $A D L)$, deteriorate quality of life, and undermine rehabilitation programs $[6,7]$. Treatment of CPSP remains a challenge because of the limited efficacy of options available and their adverse effects. Current therapeutic options include antidepressants, anticonvulsants, opioids, and neurostimulation therapy.

Botulinum neurotoxin (BoNT) has been approved worldwide for use in persons with movement disorders. Early in the use for cervical dystonia, several authors noted an analgesic effect that preceded and exceeded what would have been expected solely as a consequence of muscle relaxation [8]. Since then, several studies have been conducted to investigate its analgesic action, and in October 2010, onabotulinum toxin A was approved by the United States Food and Drug Administration for chronic migraine. Several studies and reports have suggested the efficacy of BoNT in treating neuropathic pain syndromes, including postherpetic neuralgia [2], post-traumatic/postoperative neuropathies [1], and trigeminal neuralgia [3]. We conducted a search of the scientific literature and found 2 documented cases concerning central pain and BoNT use, but both were related to treatment of central pain in spinal cord injury. No case reports were found regarding CPSP and BoNT use. In this article we report the first cases of patients with CPSP who were treated with BoNT for their spasticity and concomitantly showed a decrease in the intensity of their CPSP.
Case 1

A 48-year-old right-handed white woman with a medical history of depression experienced an ischemic stroke affecting the right cerebral hemisphere (the vascular territory of the right anterior and middle cerebral arteries). She required a right frontal decompressive craniectomy with subsequent cranioplasty. After clinical stabilization, she was admitted to an inpatient comprehensive rehabilitation program that focused on motor re-education, ADL training, and cognitive stimulation; this program lasted 3 months. She was then discharged and participated in an outpatient rehabilitation program for about a year and a half, after which she was referred to our outpatient spasticity clinic.

On clinical examination the patient presented with left spastic hemiparesis, without selected active movements in her upper or lower limbs. With regard to spasticity, the mean score on the Ashworth Scale was 2.44 in the upper limb and 1.6 in the lower limb. She also presented with left hemibody severe mechanical allodynia (rated $8 / 10$ on the numeric pain scale). She described this pain as being generated by the brushing of garments on her skin; it started 3 months after the stroke occurred and did not progress in intensity (it was graded as "severe" from the beginning). No quality of life scales or questionnaires were used, but she mentioned that these symptoms severely impaired her quality of life. She took the following oral medications: valproate, $500 \mathrm{mg}$ twice a day; agomelatine, $25 \mathrm{mg}$ every day; pregabalin, $50 \mathrm{mg}$ twice a day; atorvastatin, $20 \mathrm{mg}$ every day; acetylsalicylic acid, $150 \mathrm{mg}$ every day; omeprazole, $20 \mathrm{mg}$ every day; tizanidine, $6 \mathrm{mg}$ every day; and baclofen, $10 \mathrm{mg}$ three times a day. The patient was considered for BoNT treatment to reduce the spastic patterns of her adducted/internally rotated shoulder, flexed wrist, and clenched fist and thus, it was hoped, to improve active functionality in ADL. One week later we injected abobotulinum toxin $A$, reconstituted in $2.5 \mathrm{~mL}$ of sodium chloride $0.9 \%$ solution 
(200 U per milliliter of saline solution), under electromyographic (EMG) guidance, into the following muscles: pectoralis major $(50 \mathrm{U})$, subscapularis $(50 \mathrm{U})$, pronator teres $(25 \mathrm{U})$, flexor carpi radialis $(25 \mathrm{U})$, and flexor carpi ulnaris $(25 \mathrm{U})$. At 1 -month follow-up the patient displayed an improvement in spastic patterns (the mean score in the Ashworth Scale was 1.77 in the upper limb), as well as a reduction of her mechanical allodynia in the left upper limb (according to the numeric pain scale the intensity changed from 8 to 6) but not in the left lower limb (which remained 8 on the numeric pain scale). (We used the numeric pain scale, which has a standardized application procedure. We presented it to the patients and asked them to grade the severity of their mechanical allodynia, in the past week, considering that $0=$ no pain and $10=$ the worst imaginable pain.) The patient noticed this effect around 15 days after receiving the injection. She reported this effect as an improvement in her skin tolerance to garments, and she denied experiencing any adverse events. At the 3month follow-up, she showed recurrence of her upper limb spasticity (a mean Ashworth score in the upper limb of 2.3), but experienced a persistent reduction of the left upper limb allodynia (which remained graded as $6 / 10)$. At this time, she received another injection with BoNT for her spasticity: pectoralis major (200 U), subscapularis (100 U), latissimus dorsi (200 U), flexor carpi radialis $(100 \mathrm{U})$, flexor carpi ulnaris $(100 \mathrm{U})$, flexor digitorum sublimis (100 U), flexor digitorum profundus $(100 \mathrm{U})$, adductor pollicis $(30 \mathrm{U})$, and flexor pollicis longus $(50 \mathrm{U})$. Two months later we performed a telephone interview, and she reported an improvement in spastic patterns (we could not objectively evaluate muscle tone via the telephone). She reported that she had maintained the same magnitude of reduction of mechanical allodynia (rated 6/10). She denied experiencing any adverse events.

\section{Case 2}

A 57-year-old right-handed white man with a medical history of diabetes mellitus type 2 and primary arterial hypertension experienced a hemorrhagic stroke of the left cerebral hemisphere (frontal and parietal lobes) in 2005. Ever since, he had been integrated in an outpatient rehabilitation program and was referred to our outpatient-based spasticity clinic in 2013. On clinical examination we found global aphasia and right spastic hemiparesis with no active movements in the upper limb and poorly selective active movements in the lower limb ("en bloc" movements). With regard to spasticity, the mean score in the Ashworth Scale was 2.3 in the upper limb and 3 in the lower limb. He also presented with right hemibody mechanical allodynia of moderate intensity (rated $6 / 10$ on the numeric pain scale). No previous diagnosis had been made, nor did the caretakers seem to be aware of this condition, probably because of the patient's poor comprehension and motor fluency. Past details about allodynia (duration, course, and impact on life) were not reliable because of his poor comprehension and decreased fluency. Only simple data, such as the presence and intensity of allodynia, were reliably obtainable. He was considered for BoNT chemodenervation with the objectives of reducing the following spastic patterns: flexed elbow, clenched fist, pes equinus, and flexed toes. One week later he received an injection of incobotulinumtoxin A (diluted in $2 \mathrm{~mL}$ of normal saline solution; $50 \mathrm{U}$ per milliliter of saline solution) under EMG guidance in the following muscles: biceps brachii (100 U); brachioradialis (50 U); flexor digitorum sublimis (50 U); flexor digitorum profundus (50 U); gastrocnemius (medial head $50 \mathrm{U}$, lateral head $50 \mathrm{U}$ ); and flexor digitorum longus $(50 \mathrm{U})$. Upon re-evaluation after 1 month, he showed reduced spastic patterns (mean score in the Ashworth Scale for the muscle tone in the upper limb 1.5 and 2 in the lower limb), as well as a decrease of the mechanical allodynia intensity from 6/ 10 to $3 / 10$ on the numeric pain scale. At the 3-month follow-up he maintained the improvement in allodynia intensity (rated 3/10) but presented with an increase in muscle tone (a mean grade of 2 in the upper limb and 2.5 in the lower limb). This patient denied experiencing any adverse events.

\section{Discussion}

Patients with CPSP may evidence allodynia that can be evoked by tactile stimuli, cold, or muscle contraction [9]. The most frequent form is pure mechanical allodynia, but multiple stimulus-evoked allodynia is also possible [9]. The type, location, and intensity may depend on the location and severity of the brain lesion. In some studies, brainstem or thalamic lesions were related to higher pain intensities. Pure tactile allodynia is associated with ventrolateroposterior thalamic lesions, whereas cold allodynia tends to have more dorsally placed thalamic lesions, and movement-evoked allodynia has more anteriorly located lesions [9].

BoNT is a unique candidate for inhibition of pain transmission in CPSP. The light chain of BoNT-A functions as an endopeptidase that, in the presence of zinc, deactivates the synaptosomal-associated protein of Mr25,000 (SNAP-25) located in the cell membrane, leading to an inability of the acetylcholine vesicle to merge with the cell membrane [5]. However, SNAP-25 was also identified in sensory neurons, and the verification of its cleavage by BoNT-A led to the development of several hypothetical mechanisms of action. Recent behavioral and immunohistochemical studies indicate that the antinociceptive action occurs primarily in the central nervous system $[8,10,11]$. BoNT-A is longdistance retrogradely transported to the CNS in cholinergic axons $[9,12]$. In short, it was demonstrated in 
neuropathic pain models that a bilateral analgesic effect exists after unilateral toxin injection $[9,12]$, along with prevention of BoNT-induced antinociceptive effect by the axonal transport-blocker colchicine $[8,10,13]$. At the level of the spinal cord, in vivo experiments showed that BoNT-A inhibits the release of pain peptides, substance $P$, bradykinin, calcitonin gene-related peptide, and glutamate from the dorsal root [14].

On the other hand, using an antibody specific for BoNT-A-cleaved SNAP-25, a group of investigators discovered axonal and transynaptic transport of BoNT-A in the brain (between the 2 hippocampi) and from the periphery to the facial motor nucleus [9]), as well as to the spinal cord dorsal and ventral horns via the sciatic nerve [12]. An in vivo animal experiment revealed that BoNT-A blocks glutamate exocytosis from synaptosomes, modifying or even preventing the development of central pain by group I metabotropic glutamate receptors [15]. Another study showed that femtomolar concentrations of BoNT-A inhibit membrane $\mathrm{Na}^{+}$channels in rat central neurons, which may be implicated in central pain transmission mechanisms [15]. Specifically regarding allodynia, one experiment in diabetic rats with bilateral allodynia showed that unilateral subcutaneous injection of BoNT-A in the allodynic region of one affected limb improved allodynia in both limbs, further indicating a central analgesic effect of the toxin [11]. All these experiments suggest that retrograde axonal transport to the CNS is responsible for the antinociceptive activity of BoNT-A. It could be assumed that, in sensory areas of the CNS, BoNT-A directly or indirectly affects central neurotransmitters involved in pain processing [13].

The 2 patients we described received intramuscular injections of BoNT, and in addition to the muscle tone decrease, they presented with a somewhat unexpected and long-lasting relief of their CPSP. Oral medications, including antidepressants and anticonvulsants, did not seem to provide an acceptable analgesic effect. A limitation to the explanation for our findings is that we cannot exclude a decrease in muscle tone as the cause of the reduction of pain. Nonetheless, this does not seem to be the case, because the analgesic effect lasted longer than the muscle tone reduction. Although no decrease of the magnitude of the analgesic effect occurred during the 3-month interval between BoNT-A injections, it is possible that it lessens with time. We also recognize that the utilization of the numeric pain scale represents a limitation in itself and as the only endpoint used. More studies are necessary to further explore the analgesic effect of BoNT in CPSP and to clarify its therapeutic mechanism. Functional measures and use of other validated pain scales (e.g., the Brief Pain Inventory) would enhance the value of any future studies.

\section{Conclusion}

Increasing evidence supports the analgesic effect of BoNT, which potentially has a dual mechanism: peripheral and central. It has been studied and proved effective in relieving pain, namely in persons with chronic migraine. Our 2 stroke patients presented with CPSP and spastic hemiplegia and were treated with intramuscular injections of BoNT-A; they experienced a modest relief of their CPSP that seemed to be independent from the muscle tone reduction. More clinical research is needed to see if our findings are reproducible and to clarify the pain relief mechanism. As in other pain syndromes, BoNT may have a future role in the treatment of CPSP.

\section{References}

1. Kumar G, Soni C. Central post-stroke pain: Current evidence. J Neurol Sci 2009;284:10-17.

2. Glader E, Stegmayr B, Johansson L, Hulter-Asberg K, Wester P. Differences in long-term outcome between patients treated in stroke units and in general wards: A 2-year follow-up of stroke patients in Sweden. Stroke 2001;32:2124-2130.

3. Sulch D, Melbourn A, Perez I, Kalra L. Integrated care pathways and quality of life on a stroke rehabilitation unit. Stroke 2002;33: 358-363.

4. Jonsson A, Lindgren I, Hallstrom B, Norrving B, Lindgren A. Prevalence and intensity of pain after stroke: A population based study focusing on patients' perspectives. J Neurol Neurosurg Psychiatry 2006; 77:590-595.

5. Leijon G, Boivie J, Johansson I. Central post-stroke pain-neurological symptoms and pain characteristics. Pain 1989;36:13-25.

6. Widar M, Ahlstrom G. Disability after a stroke and the influence of long-term pain on everyday life. Scand J Caring Sci 2002;16:303-310.

7. Jensen M, Chodroff M, Dworkin R. The impact of neuropathic pain on health-related quality of life: Review and implications. Neurology 2007;68:1178-1182.

8. Ranoux D, Attal N, Morain F, Bouhassira D. Botulinum toxin type A induces direct analgesic effects in chronic neuropathic pain. Ann Neurol 2008;64:274-284.

9. Bowsher D. Allodynia in relation to lesion site in central poststroke pain. J Pain 2005;6:736-740.

10. Gonzalez G. Central pain: Diagnosis and treatment strategies. Neurology 1995;45(12 suppl 9):S11-S16, discussion S35-S36.

11. Zorowitz R, Smout R, Gassaway J, Horn S. Usage of pain medications during stroke rehabilitation: The Post-Stroke Rehabilitation Outcomes Project (PSROP). Top Stroke Rehabil 2005;12:37-49.

12. Welch M, Purkiss J, Foster K. Sensitivity of embryonic rat dorsal root ganglia neurons to Clostridium botulinum neurotoxins. Toxicon 2000;38:245-258.

13. Durham P, Cady R. Regulation of the calcitonin gene-related peptide secretion from trigeminal nerve cells by botulinum toxin type A: implications for migraine therapy. Headache 2004;44:35-42.

14. Cui M, Khanijou S, Rubino J, Aoki KR. Subcutaneous administration of botulinum toxin A reduces formalin-induced pain. Pain 2004; 107:125-133.

15. Mills C, Johnson K, Hulsebosh C. Group I metabotropic glutamate receptors in spinal cord injury: Roles in neuroprotection and the development of chronic central pain. J Neurotrauma 2002;19:23-42. 


\section{Disclosure}

A.C.-B. Spasticity Clinic/Neurophysiology Unit, Centro Hospitalar de Lisboa Central, Rua Jose Antonio Serrano, 1150-199 Lisbon, Portugal. Address correspondence to: A.C.-B.; e-mail: alexandre.camoes@gmail.com Disclosure: nothing to disclose
A.-F.N. PMR Department, Centro Hospitalar de Lisboa Central, Lisbon, Portugal Disclosure: nothing to disclose

An abstract of this article was presented at the Neurotoxins International Conference, January 14-17, 2015, Lisbon, Portugal.

Submitted for publication February 4, 2015; accepted December 2, 2015. 